\section{Anaesthesia for urological surgery}

\author{
Hazem H Alaali
}

Michael G Irwin

\begin{abstract}
Anaesthesia is commonly used to facilitate urological procedures and many patients are elderly with multiple co morbidities. Urological procedures range from minor day case to major surgery in which extensive resources are needed both intra and postoperatively. For simple day case procedures like cystoscopy or uretroscopy, general anaesthesia is most commonly used because it allows for early ambulation. Transurethral resection of the prostate (TURP) needs special attention. TURP syndrome due to excessive absorption of the irrigation fluid can be catastrophic if not managed early. Avoiding contributing factors and choosing regional anaesthesia which allows for early detection are key. Careful perioperative planning and risk stratification is important in major urological cancer surgery. Most of these procedures will require general anaesthesia (due to longer operative time and more extensive surgical trauma). Postoperative pain management in the form of epidural or patient controlled multimodal analgesia are essential. Postoperative high dependency care is beneficial.
\end{abstract}

Keywords general anaesthesia; postoperative care; preoperative assessment; regional anaesthesia; surgery; transurethral resection of prostate syndrome; urology

Introduction

Anaesthesia is commonly used to facilitate urological procedures and many patients are elderly with multiple co morbidities. Urological procedures range from minor day case procedures, to major surgery in which extensive resources are needed both intra and postoperatively (HDU-ICU). Consequently anaesthesia may encompass local, monitored anaesthesia care (MAC), regional or general techniques. ${ }^{1}$

\section{Preoperative assessment}

A thorough assessment of the patient history, including any anaesthetic records, is important in detecting any underlying co

Hazem H Alaali BSC MD CABA is a Fellow in Anaesthesiology, University of Hong Kong, Hong Kong. He is also a Chief Resident in Anaesthesia at Salmaniyah Medical Complex, Kingdom of Bahrain. Conflict of interest: none declared.

Michael G Irwin MB ChB mD fRCA fanzCA fHKam is Professor and Head, Department of Anaesthesiology, University of Hong Kong, Hong Kong, Honorary Consultant in Anaesthesia and Chief of Service of the Department of Anaesthesiology, Queen Mary Hospital, Hong Kong. Conflicts of interest: none declared.

\section{Learning objectives}

After reading this article, you should be able to:

- formulate an anaesthetic plan and analgesic regime for the management of common urological procedures

- identify the clinical manifestations of the TURP syndrome and how to manage it

- name commonly used drugs in anaesthesia with a potential for significant accumulation in patients with renal impairment or that may induce nephrotoxicity

morbidities and to assess the degree of major organ function. Exercise tolerance is a good predictor of propensity to postoperative complications and can be assessed as metabolic equivalent tasks (METS) where 1 MET is the metabolic equivalent of a resting adult. $<4$ METS or the inability to walk up 2 flights of stairs is associated with increased risk.

Special attention should be paid to the drug history and their effects on renal function.

Physical examination and lab investigations can be performed based on history and physical examination. Most perioperative medical complications involve the cardiac and \or respiratory systems so these deserve special attention. Renal function may also be compromised and many patients will require testing preoperatively.

The lithotomy position is most commonly used during urological surgeries and patient suitability for this may need to be assessed and any limitation of movement in hip/knee joints documented.

\section{Intraoperative care}

Standard American Society of Anesthesiologists (ASA) monitoring (Non-invasive blood pressure (NIBP), electrocardiograph (ECG), pulse oximetry, capnometry, temperature) should be used in all patients. Further more invasive monitoring will depend on the type of surgery and patient co-morbidity.

For obvious anatomical reasons, Urological surgery is a field where regional anaesthesia is widely practiced. Benefits of regional anaesthesia include lesser effects on the respiratory function, faster postoperative oral intake, pain relief, a lower incidence of postoperative deep vein thrombosis (DVT), and it allows easier detection of neurological symptoms during transurethral resection of the prostate (TURP) surgery (see below).

General anaesthesia (GA) is mostly chosen for very short procedures (day case cystoscopy), for lengthy major surgery (radical cystectomy, nephrectomy) or when particular positioning is required e.g. lateral or head down. It is, of course, possible to combine regional and general anaesthesia with the use of epidural techniques. This can have the advantage of reducing general anaesthetic drug requirements and can facilitate more prolonged postoperative analgesia. Epidural anaesthesia can also help reduce intraoperative blood loss and bowel distension during pelvic and intra-abdominal cancer surgery.

Renal function may be impaired and, therefore, it is prudent to choose drugs which are not likely to further compromise renal function and which are not metabolized in the kidney. Where this is not possible, then the pharmacodynamic effects must be 
closely monitored. For example muscle relaxants such as atracurium or cistracurium would be preferable to rocuronium but if there was a good reason to use rocuronium then neuromuscular function can be assessed and the effects reversed with sugammadex if necessary. Suxamethonium is best avoided in patients with renal impairment in which serum potassium may be raised.

Non-steroidal anti-inflammatory drugs (NSAID) and cyclooxygenase 2 inhibitors (coxibs) can interfere with renal autoregulation. Total intravenous anaesthesia (TIVA) with propofol and remifentanil is safe in renal dysfunction. Patients may be more sensitive to the pharmacodynamics effects in the presence of uraemia and protein binding may be reduced leading to higher free drug fractions. Consequently careful titration of drugs with target controlled infusion (TCI) is a suitable technique.

Postoperative nausea and vomiting (PONV) is not a particular problem for this type of surgery but can be reduced by good pain management (minimizing opioid use, where possible), adequate hydration and prophylactic antiemetic use.

\section{Postoperative care}

Postoperative pain management aims to provide good analgesia with little or minimal residual sedation to facilitate faster discharge. For day case procedures, short acting opioids like fentanyl along with paracetamol can be safely used.

Postoperative pain management in inpatients usually includes patient controlled analgesia (PCA) or epidurals. If PCA to be used, one should keep in mind the renal functions and the cumulative effects of opioids metabolites. Some anaesthetists advocate using fentanyl in comparison to morphine as fentanyl has no active metabolites, where morphine is metabolized by the liver to morphine 6 glucuronide (M6G) which then excreted by the kidneys. ${ }^{2}$ Drugs like paracetamol can be used safely.

\section{Endoscopic procedures of the lower genitourinary tract}

Examination of the bladder and lower genitourinary tract (GU) are very common procedures both for the diagnosis and treatment of diseases. Cystoscopy can be performed with a flexible fibrescope under local anaesthesia (lignocaine lubricant gel) with or without mild sedation. Rigid cystoscopy is indicated for the diagnosis of the lower GU pathology, and/or dilatation of urethral strictures.

General anaesthesia in the lithotomy position is most commonly used as most of these procedures are short day cases requiring early ambulation. Spinal anaesthesia may also be used and one must ensure a block to around the T10 dermatome to achieve satisfactory conditions for surgery.

\section{Transurethral resection of the prostate (TURP)}

Benign prostatic hyperplasia occurs in around $40 \%$ of men above 60 years old so, consequently, transurethral resection of the prostate is the most common surgical intervention in the urosurgical patients. During the procedure, a resectoscope is inserted through a modified cystoscope and facilitates the prostatic tissue to be cut and coagulated. Significant bleeding may occur due to opening of venous sinuses.

Various complications can develop during the procedure. Most important of these is bleeding and the TURP syndrome. Other complications may include hypothermia, bladder perforation, coagulopathy and postoperative sepsis.
Patients are usually elderly ( $>60$ years) with multiple co morbidities. Chronic renal impairment is not unusual in the elderly and where there has been significant urinary tract outflow obstruction. A detailed pre-anaesthesia check must be performed. The mortality for TURP ranges between 0.5 and $0.7 \%$ mainly due to heart failure, pulmonary oedema and renal failure. Cross matched blood should be available.

Care must be taken during patient positioning (lithotomy). Two persons are needed to move the legs simultaneously up or down to avoid stressing the spinal ligaments. Pressure points must be padded, most importantly to avoid the straps of the legs from exerting excessive pressure on contact points. Iatrogenic nerve palsies recorded before include, injury to the common peroneal nerve (loss of dorsiflexion of the foot) due to strap pressure against the head of the fibula; injury to the saphenous nerve (numbness of the medial calf) due to tight straps over the medial aspect of the legs; and injury to the obturator component of the femoral nerve due to excessive flexion of the thigh against the groin.

Unless contraindicated, regional anaesthesia (single subarachnoid injection) is recommended popular technique for TURP, as it allows early identification of the neurological manifestation of the TURP syndrome. Also the vasodilatation produced and the venous pooling helps reduce the circulatory overload, and decreases the incidence of postoperative (DVT). If GA is chosen, it is best achieved with controlled ventilation via a supraglottic airway device. High airway pressures must be avoided as this increases bleeding from the prostatic bed. A large calibre intravenous cannula is sited in case of significant bleeding. A forced air warmer, fluid warmer and body temperature irrigation fluids should be used to maintain normothermia.

\section{TURP syndrome}

TURP syndrome refers to the symptoms and signs that occur as a result of the absorption of large amounts of irrigation fluid. It can present either intra or postoperatively. Prompt recognition and treatment is essential to limit morbidity and mortality associated with this condition. ${ }^{3}$

Many factors contribute to the development of TURP syndrome. The type of irrigation fluid is shown in Table 1; (surgery should $\mathbf{Q 3}$ ideally be less than $1 \mathrm{~h}$ ); the hydrostatic pressure of the irrigation fluid should be $<60 \mathrm{~cm}$ above the heart level; and the prostatic venous pressure should not be low (maintain normovolaemia).

The average amount of irrigation fluid absorbed is $20 \mathrm{ml} \backslash \mathrm{min}$ (1-1.5 $\mathrm{L}$ during the average procedure $(1 \mathrm{~h}))$, but this may increase if the precautions listed above not taken into consideration.

Ideal irrigation fluid

Isotonic (prevent haemolysis)

Electrically inert (prevent current dissipation from diathermy)

Transparent (no impairment of surgical field visualization)

Sterile (prevent infection)

Inexpensive

No ideal irrigation solution exists. Most commonly used solutions are $1.5 \%$ glycine (288 mmol/L), $2.7 \%$ sorbitol (195 mmol/ $\mathrm{L})$ or $3 \%$ mannitol. Among them, glycine is most commonly used. Glycine is a non-essential amino acid, metabolized in the 
Commonly used drugs in anaesthesia with a potential for significant accumulation in patients with renal impairment

$\begin{array}{ll}\text { Muscle relaxants } & \begin{array}{l}\text { Rocronium, vecronium } \\ \text { Pnalgesics }\end{array} \\ \text { Induction agents } & \begin{array}{l}\text { Barbiturates } \\ \text { Atropine, glycopyrolate } \\ \text { Anticholinergics } \\ \text { Cholinesterase }\end{array} \\ \text { inhibitors } & \text { Neostigmine, edrophonium } \\ \text { Antibiotics } & \begin{array}{l}\text { Penicillins, cephalosporines, } \\ \text { aminoglycosides, } \\ \text { vancomycin } \\ \text { Clonidine, methyldopa, } \\ \text { Antihypertensives }\end{array} \\ \text { Miscellaneous } & \text { Inotropes, digoxins }\end{array}$

\section{Table 1}

liver to ammonia. It has some cardiac and CNS toxicity and is an inhibitory neurotransmitter in the retina. A large amount of glycine slows down the transmission of impulses from the retina to the cerebral cortex causing transient blindness in some cases (resolves spontaneously within $24 \mathrm{~h}$ ).

The use of glycine solution with a tracer amount of ethanol for detecting and quantifying irrigating fluid absorption has been pioneered by Hahn and his colleagues in Sweden. ${ }^{4}$ Alcometers can be used easily during both general anaesthesia and regional to help in early detection of glycine toxicity.

During regional anaesthesia, the patients may complain of nausea, chest tightness, shortness of breath, dizziness, restlessness, confusion and blurring of vision. These signs will, of course, be masked by general anaesthesia and diagnosis delayed. Patients may have an increase or decrease in blood pressure with refractory bradycardia. On the ECG nodal rhythm, ST segment changes, $\mathrm{U}$ waves and widening of the QRS complexes may be observed.

The best way to manage TURP syndrome is to prevent it. Avoiding contributing factors (see above) and choosing regional anaesthesia which allows for early detection are important.

\section{Management of TURP syndrome}

Notify the surgeon

Stop surgery after coagulating bleeding points

Airway, Breathing, Circulation

Supplementary oxygen and ventilate if needed

Fluid restriction

Check urea, electrolytes, haemoglobin and arterial blood

gas 2 semide $20 \mathrm{mg}$ iv

Mild hyponatraemia $(\mathrm{Na}>120)$, fluid restriction may suffice

Severe hyponatraemia $(\mathrm{Na}<120)$, use hypertonic saline

Slow correction ( $<0.5 \mathrm{mmol} /$ hour), to avoid central

pontine myelinosis

Seizures: anticonvulsant e.g. midazolam,

diazepam or thiopentone

Hypotension: inotropic support and invasive monitoring

Admit to intensive care unit postoperatively

\section{Laser prostatectomy}

A laser can be used to destroy the enlarged prostate tissue as a minimally invasive procedure and an alternative to TURP. A Cochrane review ${ }^{5}$ of 20 studies involving 1898 subjects found laser techniques to be useful and relatively safe alternatives to TURP. The small number of enrolled subjects and differences in study design limit any definitive conclusions regarding which type of laser technique is the most effective. Improvements in symptoms and urine flow slightly favoured TURP, though laser procedures had fewer side effects and shorter hospitalization times. The follow-up durations of these studies ranged from 6 to 36 months and men with extremely large prostates were generally excluded from the trials. The risk of needing a reoperation for recurrent symptoms was higher following laser procedures. Study results were insufficient to adequately compare laser techniques with other minimally invasive procedures. More studies, using randomized treatment assignment, enrolling larger numbers of subjects, and comprehensive measures of treatment effectiveness and side events, are needed to better define the long-term safety and durability of laser techniques for treating LUTS associated BPO.

The anaesthetic management of laser prostatectomy is similar to TURP, with the benefit of less fluid absorption so that the risk of TURP syndrome is reduced. Also, the precise laser beam causes less bleeding compared to the ordinary resectoscope.

\section{Endoscopic procedures of the upper genitourinary tract}

Ureteroscopy is used to outline the anatomy of the upper urinary tract and kidneys, remove renal calculi, stent the ureters and for renal biopsies.

Most of these patients have renal impairment secondary to genitourinary tract obstruction, so renal function should be checked.

Generally these are done as day case procedures in the lithotomy position under general anaesthesia without muscle relaxation (often using a LMA). Postoperative pain is usually mild and can be easily managed with simple analgesics such as paracetamol. As usual, NSAIDs will be contraindicated in the presence of renal insufficiency.

\section{Percutaneous stone removal}

This is indicated for the removal of large (staghorn) stones. Patients are usually healthy but may have had haematuria which may require a full blood count check if severe. As before, renal obstruction can result in impairment of function.

The procedure first involves stenting of the ureter (in the lithotomy position), then the patient is placed prone to insert a guide wire percutaneously into the renal pelvis to conduct the nephroscope.

Due to the prone position, general anaesthesia with the use of a reinforced endotracheal tube is a suitable technique. Special attention should be paid to appropriate protection and padding for pressure points such as the eyes, nose and nerves, and keeping the head in the neutral position. It is also important to document that such measures have been taken. The chest and pelvis must be supported while the abdomen is free.

Postoperative pain can be managed with opioids and/or paracetamol. Again, NSAIDs are avoided if there is impaired renal 
function. A chest radiograph can be ordered if there is a suspicion of pneumothorax.

\section{Major (radical) surgery}

There is a wide variety of major urological procedures performed, mainly for the treatment of tumours of the genitourinary tract. Such surgery requires careful perioperative planning and risk stratification in order to formulate a management strategy.

The main concerns are long operative time, fluid and blood loss with consequent possible blood transfusion, large surgical incision and the need for more aggressive pain management. This is coupled with a generally elderly patient population and a more marked surgical stress response. Most of these procedures will require general anaesthesia (due to longer operative time and more extensive surgical trauma). Contemporaneous epidural analgesia is a useful addition as it reduces intraoperative anaesthetic drug requirements, reduces respiratory complications, facilitates weaning and produces excellent postoperative analgesia. Patient controlled multimodal analgesia is an acceptable alternative. Cross matching of enough blood units and maintaining normothermia with fluid and forced air warming devices is mandatory. As most surgery is neoplastic, cell saver devices are seldom used. A large bore intravenous cannula is inserted and invasive monitoring such as arterial and/or central venous line insertion can be considered depending on the extent of surgery and co-morbidity. Point of care testing for arterial blood gases and blood parameters ( $\mathrm{Hb}$, electrolytes, and coagulation) can be useful. Prearrangement of a high dependency or intensive care unit bed is advisable.

\section{Radical prostatectomy}

Prostatic carcinoma is the most commonly diagnosed cancer in men and is present in $75 \%$ over 75 years old and. Radical prostatectomy is indicated under the age of 70 years with localized disease and who have a life expectancy of at least 10 years. As the name implies, it involves removing the whole prostate gland, the seminal vesicles, the ejaculatory ducts and a portion of the bladder neck. The patient is placed in the hyperextended supine position with the iliac crest over the break in the operating table. Care must be taken not to put excessive strain on the back. The OR table is also tilted head down to make the operative field horizontal.

Precautions for rapid fluid and blood administration must be in place. Epidural analgesia is useful as described above.

Many surgeons will combine radical prostatectomy with laparoscopic pelvic lymph node dissection. Here there are added concerns. Often a steep head down trendelenburg position and rotation from side to side is needed to assist surgical exposure. There is a risk of $\mathrm{CO}_{2}$ absorption from the peritoneum. The surgeon may ask for injection of a dye (indigo carmine to visualize the ureters) and this can cause changes in the blood pressure. As surgery is prolonged with high intra-abdominal pressures and involves the pelvic region, DVT prophylaxis must be ensured.

Many surgeons will combine radical prostatectomy with laparoscopic pelvic lymph node dissection. Here there are added concerns. Often a steep head down trendelenburg position and rotation from side to side is needed to assist surgical exposure. There is a risk of $\mathrm{CO}_{2}$ absorption from the peritoneum. The surgeon may ask for injection of a dye (indigo carmine to visualize the ureters) and this can cause changes in the blood pressure. As surgery is prolonged with high intra-abdominal pressures and involves the pelvic region, DVT prophylaxis must be ensured.

There has been growing interest in the last few years in laparoscopic and robot assisted prostatectomy. These procedures are less invasive and produce a quicker recovery and return to daily activities. The blood loss is markedly reduced especially with robotic surgery. The anaesthetic implications are similar to other laparoscopic surgical procedures, although the operations can be quite long and may necessitate a prolonged time in the head down, trendelenburg position. Patients can be managed postoperatively in the general ward.

\section{Nephrectomy}

Nephrectomy is a major procedure performed to remove renal tumours or non-functioning kidneys. It may also be used to retrieve healthy kidneys for living related organ donation. Apart from the healthy kidney donors, most of patients presenting for nephrectomy have impaired renal function. Some may have a paramalignant syndrome such as polycythaemia, hypercalcaemia or hypertension. General anaesthesia is usually required because of the long operative time as well as patient discomfort from the position. Most nephrectomies are performed in the modified lateral position with a kidney rest under the iliac crest (Figure 1). The loin is hyperextended over the table break to open the flank space, and the upper arm is suspended above the patient. It is important to ensure that arms are not overstretched. Potential intraoperative complications include massive bleeding, hypotension due to the position causing retraction of the IVC, and pneumothorax due to opening of the pleura during surgery. Occasionally the tumour may involve the IVC necessitating the clamping leading to hypotension. In rare cases of extensive tumour extension, thoracotomy may be needed, while very rare cases where IVC thrombosis has spread to the heart, cardiopulmonary bypass may even be needed. Epidural analgesia is recommended in radical nephrectomy. Other options include a paravertebral block (single shot or continuous via a catheter), PCA and/or regular paracetamol. NSAIDS are generally avoided

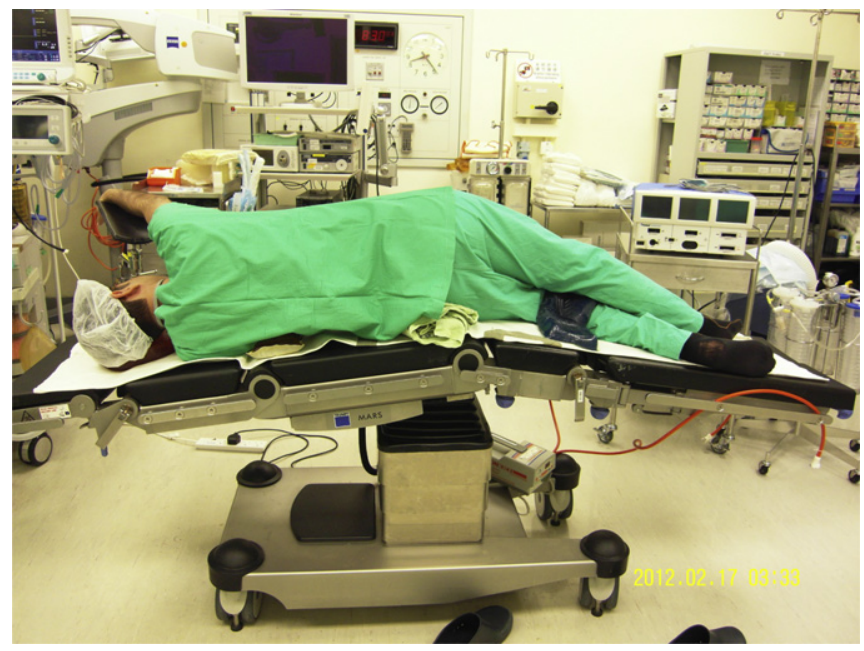

Figure 1 Modified lateral (hyperextended) position using a kidney rest under the iliac crest to improve the exposure of the flanks. 
due to the poor renal reserve in these patients and potential for bleeding.

Laparoscopic nephrectomy has become the standard of care now in many places for removal of small sized kidney tumours. Also, laparoscopic donor nephrectomy is increasingly becoming the preferred method in kidney retrieval. ${ }^{6}$ The procedure requires the patient to be in the lateral decubitus position with intraabdominal insufflation of $\mathrm{CO}_{2}$ and its attendant effects. Major complications include sepsis, bowel injury, rhabdomyolysis and acute renal failure. The procedure may be converted to open and the anaesthetist should explain this to the patient beforehand and have a clear plan should this eventuate.

\section{Radical cystectomy}

This involves excising the urinary bladder and forming a urinary conduit, usually ileal or colonic, to direct the flow of urine. In men, surgeons may have to remove the seminal vesicles and proximal urethra while in women excising the anterior vaginal wall and uterus may be included. Cigarette smoking is a risk factor for bladder carcinoma, ischaemic heart disease and chronic obstructive lung disease are common co morbidities. Many patients may have already undergone chemotherapy. Preoperative assessment should identify any adverse effects of the cytotoxic drugs (e.g. bone marrow depression, renal impairment with cisplatin, pulmonary fibrosis with bleomycin, neuropathy from vincristine).

General anaesthesia with epidural analgesia is recommended. As with other radical surgeries, invasive monitoring may be useful.

Mannitol can be given before dissection near to the renal vessels to help reduce ischaemic renal injury (secondary to renal vasospasm). During urinary diversion to the ileum, it will no longer be possible to measure the urine output. Postoperatively, the most commonly encountered complication is hyperchloraemic metabolic acidosis and hypokalaemia secondary to urinary absorption from the GI tract. Ileus is very common and nasogastric drainage may be helpful.

\section{Minor urological procedures}

Circumcision: in babies younger than 3 months, it can be performed as day case surgery under local anaesthesia in the form of a dorsal penile nerve block or caudal epidural block. ${ }^{7}$ Older children usually require general anaesthesia with a block for postoperative pain management with paracetamol or NSAIDs as required.

Orchidectomy: is indicated in case of injury, torsion or to treat testicular carcinoma. General anaesthesia with supraglottic airway can be chosen. Supplemental ilioinguinal block may be performed to help reduce the need for postoperative pain medications.

\section{REFERENCES}

1 Sabaté $\mathrm{S}$, et al. Anesthesia for urological surgery in a European region with 6.7 million inhabitants (Catalonia, Spain). J Clin Anesth 2009; 21: $30-7$.

2 Kilpatrick Gavin J, Smith Terry W. Morphine-6-glucuronide: actions and mechanisms. Med Res Rev 2005; 25: 521-44.

3 Vijayan Senthilkumar. TURP syndrome. Trends Anaesth Critic Care 2011; 1: 46-50.

4 Hanh RG. Fluid absorption in endoscopic surgery. BJA 2006; 96: 8-20.

5 Hoffman RM, MacDonald R, Wilt T. Laser prostatectomy for benign prostatic obstruction. Cochrane Database Syst Rev 2000; doi: 10.1002/14651858.CD001987.pub2. Issue 1. Art. No.: CD001987.

6 Kapoor A, Lambe S, Kling AL, Piercey KR, Whelan PJ. Outcomes of laparoscopic donor nephrectomy in the presence of multiple renal arteries. Urol Ann 2011; 3: 62-5.

7 Johr M, Berger TM. Caudal blocks. Pediatr Anesth 2012; 22: 44-50. 